\title{
Utility of percutaneous radiofrequency ablation alone or combined with transarterial chemoembolization for early hepatocellular carcinoma
}

\author{
TSUTOMU TAMAI ${ }^{1}$, AKIHIKO OSHIGE ${ }^{2}$, KAZUAKI TABU $^{1}$, ERIKO TABU ${ }^{1}$, SYO IJYUIN $^{1}$, HARUKA SAKAE $^{1}$, \\ HIROKA ONISHI $^{1}$, KAORI MUROMACHI ${ }^{1}$, AKIKO SAISYOJI ${ }^{3}$, KOHEI ODA ${ }^{1}$, KOTARO KUMAGAI ${ }^{1}$, \\ SEIICHI MAWATARI ${ }^{1}$, AKIHIRO MORIUCHI ${ }^{1}$, KAZUHIRO SAKURAI $^{3}$, TSUYOSHI HORI ${ }^{2}$ and AKIO IDO ${ }^{1}$ \\ ${ }^{1}$ Digestive and Lifestyle Diseases, Department of Human and Environmental Sciences, \\ Kagoshima University Graduate School of Medical and Dental Sciences, Kagoshima 890-8520; \\ ${ }^{2}$ Department of Gastroenterology and Hepatology, Kagoshima City Hospital, Kagoshima 890-8760; \\ ${ }^{3}$ Department of Hepatology, Kagoshima Teishin Hospital, Kagoshima 890-8798, Japan
}

Received July 19, 2016; Accepted March 30, 2017

DOI: $10.3892 / 01.2017 .6476$

\begin{abstract}
Percutaneous radiofrequency ablation (RFA) combined with transarterial chemoembolization (TACE) is an effective, standard therapy against small hepatocellular carcinoma (HCC). However, there is debate regarding the effectiveness of RFA combined with TACE (RFA/TACE) compared with RFA alone. These two approaches were compared for the treatment of early HCC. The present study examined 83 HCC tumors in 83 patients treated with RFA between April 2007 and August 2014 at three medical institutions. All HCCs were single hypervascular tumors, with a median diameter of $16 \mathrm{~mm}$ (range, 6-30 mm). The overall survival (OS) rate of all patients $(n=83)$ was $97.5 \%$ at 1 year, $82.8 \%$ at 3 years and $48.6 \%$ at 5 years, and the local recurrence rate of all patients was $14.3 \%$ at 1 year, $32.3 \%$ at 3 years and $36.5 \%$ at 5 years. The tumor-free survival (TFS) rate of all patients was $95.1 \%$ at 1 year, $56.3 \%$ at 3 years and $23.4 \%$ at 5 years. Compared with RFA alone, RFA/TACE significantly improved OS $(\mathrm{P}<0.001)$, intrahepatic distant recurrence (IDR; $\mathrm{P}=0.038)$ and TFS $(\mathrm{P}=0.010)$. A univariate analysis of prognostic indicators revealed that age $<70$ years $(\mathrm{P}=0.008)$, aspartate transaminase $<40 \mathrm{IU} / 1(\mathrm{P}=0.003)$, alanine aminotransferase $<40 \mathrm{IU} / 1$ $(\mathrm{P}=0.006)$ and platelet count $>10 \times 10^{4} / \mu 1(\mathrm{P}=0.05)$ were associated with a high survival rate. Multivariate analysis identified RFA/TACE [hazard ratio (HR), 0.108; $\mathrm{P}=0.001$ ] as an independent prognostic indicator. RFA/TACE was identified as
\end{abstract}

Correspondence to: Dr Tsutomu Tamai, Digestive and Lifestyle Diseases, Department of Human and Environmental Sciences, Kagoshima University Graduate School of Medical and Dental Sciences, 8-35-1 Sakuragaoka, Kagoshima 890-8520, Japan E-mail: tamaitsu@m.kufm.kagoshima-u.ac.jp

Key words: radiofrequency ablation, transarterial chemoembolization, early hepatocellular carcinoma, overall survival, tumor-free survival the only independent indicator of IDR (HR: 0.467; $\mathrm{P}=0.042$ ) and TFS (HR: 0.452; $\mathrm{P}=0.012$ ). RFA/TACE improved OS rate, IDR and TFS compared with RFA alone. The data suggested that RFA/TACE should be considered for the treatment of single hypervascular HCC.

\section{Introduction}

In 2012, there were 782,500 new cases of hepatocellular carcinoma (HCC) and $>745,500$ liver cancer-associated mortalities (1). Liver cancer is the second most common cause of cancer-associated mortality worldwide and has a poor prognosis (mortality/incidence ratio, 0.95) (1). There are a variety of treatment guidelines for liver cancer depending on tumor stage (2-4), and at present, liver resection and localized treatment [percutaneous ethanol injection surgery or radiofrequency ablation (RFA)] are recommended as curative and localized treatments for early-stage liver cancer (2-4). However, a number of patients harbor chronic hepatitis $\mathrm{B}$ and $\mathrm{C}$ viral infections or cirrhosis in addition to liver cancer (5). In these patients, who have decreased liver function, less invasive and more effective early treatment of liver cancer may be beneficial. Surgical resection is considered to be the only potentially curative therapy for HCC (6), but it is a highly invasive procedure. In contrast, the less-invasive percutaneous RFA is a standardized and widely used treatment method, which has equal efficacy to liver resection in terms of localized control (7). However, RFA monotherapy may increase the risk of relapse in cases where the HCC is comparatively large, when it exists near the surface of the liver or near vessels that are hard to treat due to the risk of coagulation necrosis (8). A variety of methods, including imaging support such as Real-Time Virtual Sonography (9), have been devised in the past to counteract these difficulties.

Transarterial chemoembolization (TACE) is often used prior to RFA in the treatment of early-phase HCC (10). However, the rate of local and ectopic recurrence and the long-term effect on prognosis have not been adequately investigated for this combination. The present study examined the 
long-term effectiveness of RFA/TACE compared with RFA alone.

\section{Materials and methods}

Patients. The present, retrospective study initially enrolled 192 patients, the median age was 72.0 years old (range 45-91 years old) and $47.7 \%$ were female, with a total of 283 HCC tumors treated with RFA between April 2007 and August 2014 at Kagoshima University Medical and Dental Hospital (Kagoshima, Japan), Kagoshima Teishin Hospital (Kagoshima, Japan) and Kagoshima City Hospital (Kagoshima, Japan). Among these patients, 83 patients who met the following inclusion criteria were selected for final analysis: Solitary HCC nodules $\leq 30 \mathrm{~mm}$ in diameter; strong contrast compared with surrounding liver parenchyma in early-phase dynamic contrast-enhanced computed tomography (CT) and low-density areas in the late phase; no imaging evidence of tumor invasion into the major portal or hepatic vein branches; no extrahepatic metastasis; a platelet count of $>30,000 \times 10^{4} / \mu 1$; and post-treatment observation for $\geq 3$ months.

The informed consent was waived due to the retrospective nature of the study. The study protocol conformed to the ethical guidelines of the World Medical Association Declaration of Helsinki and was approved by the Ethics Committees of Kagoshima University Medical and Dental Hospital, Kagoshima Teishin Hospital and Kagoshima City Hospital (Kagoshima, Japan).

Diagnosis of HCC. In all patients, HCC was diagnosed based on typical results by two or more imaging modalities [ultrasonography (US), CT and magnetic resonance imaging (MRI)] and characteristic serum levels of $\alpha$-fetoprotein (AFP) and des- $\gamma$-carboxy prothrombin (DCP; also termed PIVKA-II). Abdominal US was performed with a real-time scanner using a 3.5-MHz transducer (HI VISION 900S; Hitachi, Ltd., Tokyo, Japan). The US diagnosis of HCC was based on the presence of lesions with different echogenicity (hypoechoic, hyperechoic, isoechoic or a mixed pattern) compared with the surrounding liver parenchyma. Dynamic CT was performed with a multi-detector row scanner (Aquilion PRIME; Toshiba Medical Systems Corporation, Tokyo, Japan). Non-enhanced CT scans were obtained first, followed by quadra-phase contiguous CT scans with $5 \mathrm{~mm}$-thick sections. A bolus injection of $100 \mathrm{ml} \mathrm{65 \%} \mathrm{iopamidol} \mathrm{(Iomeron} \mathrm{350;} \mathrm{Eisai} \mathrm{Co.,} \mathrm{Ltd.,}$ Tokyo, Japan) was then administered at a rate of $3 \mathrm{ml} / \mathrm{sec}$. Arterial-phase CT scans were obtained at $30 \mathrm{sec}$, portal-phase CT scans were obtained at $60 \mathrm{sec}$ and late-phase CT scans were obtained at 90-120 sec. A radiologist diagnosed the CT results. The CT diagnosis of HCC was based on the presence of an enhancing lesion on arterial-phase CT scans and hypoattenuation on late-phase CT scans. AFP and DCP were performed within the week prior to RFA. Normal limits were defined as $<10 \mathrm{ng} / \mathrm{ml}$ for AFP and $<40 \mathrm{mAU} / \mathrm{ml}$ for DCP. The term 'early HCC' has two meanings, namely clinical early $\mathrm{HCC}$ and histopathological early HCC. However, in the present study it was used to mean histopathological early HCC. The Barcelona Clinic Liver Cancer (BCLC) staging system was followed $(11,12)$, which is commonly used in the United States and Europe, and early HCC was diagnosed as presenting with hypervascularity in the arterial phase by contrast-enhanced CT and classification as single-site Stage A (early stage). Patients with multiple existing HCCs and single-site lesions $>5 \mathrm{~cm}$ were excluded, according to guidelines on The Japan Society of Hepatology (4).

Treatment protocol. The treatment selection of RFA/TACE (with TACE performed prior to RFA) or RFA monotherapy was performed by specialists in HCC treatment (such as RFA and TACE) at Kagoshima University Medical and Dental Hospital, Kagoshima Teishin Hospital and Kagoshima City Hospital, according to the age, performance status (PS), liver function, tumor size and tumor location of patients.

TACE and RFA combination therapy. In the RFA/TACE group, TACE was first performed using the Seldinger technique (13) according to the following protocol. Subsequent to introducing a 3.5- or 4-Fr long sheath (Medikit Super Sheath; Medikit Co., Ltd., Tokyo, Japan) into the femoral artery, a 3.5- or 4-Fr pre-shaped catheter (Selecon-PA Catheter; Terumo Clinical Supply Co., Ltd., Gifu, Japan) was inserted into a superior mesenteric artery and 30-40 $\mathrm{ml} \mathrm{50 \%} \mathrm{iopamidol} \mathrm{(Iomeron} \mathrm{350;}$ Eisai Co., Ltd.) was injected. Computed tomography during arterial-portography (CTAP) was performed to determine whether there were one or more HCC lesions, and to assess the patency of the portal vein. Computed tomography during arteriography (CTA) was then performed to detect $\mathrm{HCC} ; 15-20 \mathrm{ml}$ of $50 \%$ iopamidol was injected via the same catheter placed in a common hepatic artery. In addition, a 2-Fr microcatheter was selectively placed in the tumor-bearing artery of the HCC (nutrient artery), and an emulsified formulation of iodized oil (Lipiodol; Laboratoire Guerbet, AulnaySous-Bois, France) was injected along with the following three anticancer agents: $20 \mathrm{mg}$ epirubicin hydrochloride (Farmorubicin; Pfizer Japan, Inc., Tokyo, Japan) and $4 \mathrm{mg}$ mitomycin C (Kyowa Hakko Kirin Co., Ltd., Tokyo, Japan); miriplatin hydrate (Miripla; Dainippon Sumitomo Pharma Co., Ltd., Tokyo, Japan); and cisplatin (Nihon-Kayaku Co., Ltd., Tokyo, Japan). Following injection of the emulsified formulation, gelatin sponge particles (Gelpart; Nippon Kayaku, Tokyo, Japan) were injected as an embolus into the same location. Hepatic arteriography was performed following the embolus injection to confirm the loss of blood flow to the tumor through the nutrient artery prior to performing the surgery. The timing of RFA following TACE varied according to the onset of side effects, overall patient condition and degree of post-operative liver dysfunction, but usually occurred within the week following TACE. RFA was performed by locally anesthetizing the injection site using $1 \%$ xylocaine (AstraZeneca, Tokyo, Japan) and the liver surface was assessed by ultrasound. A 17-G internally-cooled electrode with a 2 or $3 \mathrm{~cm}$ exposed tip (Radionics, Inc., Burlington, MA, USA) was then guided to the HCC via ultrasound for the ablation. An abdominal CT was performed 3-4 days post-RFA and the RFA treatment effect, in particular the tumor and cauterization margins, was evaluated. Treatment response was evaluated by dynamic CT within 1 week. When HCC remained evident, additional ablation was performed. RFA was performed so that margins of $\geq 5 \mathrm{~mm}$ were obtained in all patients, and additional RFA was performed where possible when the ablation area was insufficient. Patients with margins 
of 0-5 mm were classified into those where it was possible to widen the ablation area with additional RFA and those for whom additional RFA would be difficult due to the radiator effect of surrounding blood vessels or the original location of the lesion. For patients where additional RFA was not expected to return benefits, follow-up observations were performed. Patients with almost complete ablation with certain lesions with margins $<5 \mathrm{~mm}$ were included in the $0-5 \mathrm{~mm}$ margin group. All patients underwent two sessions or fewer.

RFA alone. RFA monotherapy was conducted within 1 week of hospital admission using the exact procedure aforementioned. As with the combined therapy, an abdominal CT was performed 3-4 days post-RFA and the tumor and cauterization margins were evaluated along with the RFA treatment effect. In the RFA alone group, well-differentiated HCC was excluded, as well as HCC of other sites confirmed by prior abdominal ultrasonography, gadolinium-ethoxybenzyl-diethylenetriamine pentaacetic acid-MRI, CTAP or CTA.

Clinical characteristics and laboratory markers of patients. The clinical characteristics and laboratory markers of patients assessed included age, sex, tumor size, observation period, number of RFA sessions, TACE, RFA/TACE, previous treatment and virus markers, including hepatitis $B$ virus (HBV), hepatitis $\mathrm{C}$ virus (HCV) and $\mathrm{NBNC}\left(\mathrm{HBV}^{-}\right.$and $\left.\mathrm{HCV}^{-}\right)$. Hepatic function was assessed using the Child-Pugh classification (14) based on clinical (ascites and encephalopathy) and laboratory (serum albumin, total bilirubin and prothrombin time) parameters, body mass index, aspartate transaminase (AST), alanine aminotransferase (ALT), $\gamma$-glutamyl transpeptidase $(\gamma$-GTP), serum albumin, total bilirubin, prothrombin time, platelets, AFP and DCP.

Comparison. Overall survival (OS) rates were compared using the time from the beginning of treatment to the last follow-up CT examination or mortality. RFA of a single HCC may still result in multiple recurrences and progression to the intermediate stage during follow-up. Post-treatment TACE may be performed for intermediate-stage $\mathrm{HCC}$, but this was not confirmed in the present study. Local recurrence was defined as the presence of one or more recurrent lesions within the RFA-ablated area. A patient who presented with HCC adjacent to the site of ablation after several years was excluded, and was not classified as having local recurrence. Local recurrence rates in each nodule were compared using the time from the beginning of treatment to the last follow-up CT examination. The same period was used to compare the intrahepatic distant recurrence rate, including multicentric occurrences and intrahepatic metastases in each nodule. Comparison of tumor-free survival rates among patients was conducted using the time from the beginning of treatment to local tumor progression, progression of other tumors at the last follow-up CT examination or mortality. Naïve and recurrent patients were examined together in the present study, since the two types of patients exhibited similar trends (data not shown). The present study included patients with early HCC, defined at the time of treatment based on the earliest stage according to each of the following classification systems: Tumor-node-metastasis stage (15); Child-Pugh grade (14); Japan integrated staging score (4); and the Cancer of
Table I. Clinical characteristics of patients with early, solitary and hypervascular hepatocellular carcinoma.

\begin{tabular}{lc}
\hline Characteristic & $\begin{array}{c}\text { Number of patients/ } \\
\text { mean } \pm \mathrm{SD}\end{array}$ \\
\hline Age, years & $71.6 \pm 9.2$ \\
Sex, male/female & $41 / 42$ \\
Tumor size, mm & $17.2 \pm 5.5$ \\
Observation period, months & $17.2 \pm 5.5$ \\
Number of RFA sessions & $1.2 \pm 0.4$ \\
RFA/TACE, -/+ & $27 / 56$ \\
Previous treatment, $/+$ & $41 / 42$ \\
Virus marker, HBV/HCV/NBNC & $7 / 60 / 16$ \\
Child-Pugh classification, A/B/C & $73 / 10 / 0$ \\
BMI, kg/m ${ }^{2}$ & $24.1 \pm 3.4$ \\
Biochemical analysis & \\
AST, IU/l & $49.9 \pm 21.2$ \\
ALT, IU/l & $39.8 \pm 22$ \\
$\gamma-G T P$, IU/l & $24.1 \pm 3.4$ \\
Serum albumin, g/dl & $3.6 \pm 0.5$ \\
Total bilirubin, mg/dl & $1.1 \pm 0.5$ \\
Prothrombin time, $\%$ & $81.5 \pm 14.5$ \\
Platelets, x10 $/ \mu 1$ & $10.2 \pm 4.8$ \\
AFP, ng/ml & $277.7 \pm 1,289.9$ \\
DCP, mAU/ml & $419.6 \pm 1,474.4$ \\
\hline
\end{tabular}

SD, standard deviation; RFA, radiofrequency ablation; TACE, transarterial chemoembolization; RFA/TACE, RFA combined with TACE; $\mathrm{HBV}$, hepatitis B virus; $\mathrm{HCV}$, hepatitis C virus; NBNC, HBV (-) and HCV (-); BMI, body mass index; AST, aspartate aminotransferase; ALT, alanine aminotransferase; $\gamma$-GTP, $\gamma$-glutamyl transpeptidase; AFP, $\alpha$-fetoprotein; DCP, des- $\gamma$-carboxy prothrombin.

the Liver Italian Program score (16). There were also a number of confounding factors, including tumor size, number and presence of extrahepatic metastasis. Thus, prognostic factors were not analyzed using these items.

Statistical analysis. Statistical analyses were performed using the $\chi^{2}$-test or the Mann-Whitney U test, as appropriate. The Kaplan-Meier method was used to estimate cumulative survival and progression of local and other tumors, and these distribution curves were compared using the log-rank test. Univariate and multivariate analyses of patient outcome risk ratios were performed using Cox's proportional hazards model. All statistical analyses were conducted using IBM SPSS Statistics version 21.0 (IBM SPSS, Armonk, NY, USA). Results were expressed as the median, with minimum and maximum values. $\mathrm{P}<0.05$ was considered to indicate a statistically significant difference.

\section{Results}

Characteristics of patients. A total of 83 patients met the aforementioned inclusion criteria. Table I summarizes the baseline clinical characteristics of the 83 patients with early, solitary and hypervascular HCC. 
Rate of OS, local recurrence and tumor-free survival (TFS) in all 83 patients. The OS rates of all patients during the follow-up period were $97.5 \%$ at 1 year, $82.8 \%$ at 3 years and $48.6 \%$ at 5 years (Fig. 1), and the local recurrence rates of all patients $(n=83)$ were $14.3 \%$ at 1 year, $32.3 \%$ at 3 years and $36.5 \%$ at 5 years (Fig. 2). The TFS rates of all patients $(\mathrm{n}=83)$ were $95.1 \%$ at 1 year, $56.3 \%$ at 3 years and $23.4 \%$ at 5 years (Fig. 3).

RFA/TACE compared with RFA alone. A total of 56 patients with 56 HCC nodules were treated with RFA/TACE, while 27 patients with $27 \mathrm{HCC}$ nodules were treated with RFA alone. Table II summarizes the following baseline clinical characteristics of the two groups with early, solitary and hypervascular HCC, stratified by treatment categories: Age; sex; virus markers, including HBV, HCV and NBNC; clinical laboratory parameters, including prothrombin time, bilirubin, serum albumin, AST, ALT, $\gamma$-GTP, platelets, AFP and DCP; previous treatment; intrahepatic recurrence; tumor size and ablated size. A significant difference was observed only in tumor size $(\mathrm{P}=0.004$; Table II). No further statistically significant differences were observed between the two groups.

OS rate. During the follow-up period, the cumulative survival rate of patients treated with RFA/TACE was significantly improved compared with that of patients treated with RFA alone $(\mathrm{P}<0.001$; Fig. 4).

Intrahepatic distant recurrence rate. During the follow-up period, the intrahepatic distant recurrence rate in patients treated with RFA/TACE was significantly improved compared with patients treated with RFA alone ( $\mathrm{P}=0.038$; Fig. 5). No significant differences were observed in the site of recurrence between the two groups (data not shown).

TFS rate. During the follow-up period, the TFS rate of patients treated with RFA/TACE was significantly improved compared with that of patients treated with RFA alone ( $\mathrm{P}=0.01$; Fig. 6).

Univariate analysis and multivariate statistics for OS rate. A univariate analysis using the log-rank test revealed that the survival rate varied significantly with age, AST, ALT, platelet count (Plt) and RFA/TACE (Table III). Multivariate analysis using a Cox proportional hazard model for four of these markers (AST, ALT, Plt and RFA/TACE), as well as age and sex, revealed that RFA/TACE was the only independent risk factor associated with good patient prognosis [hazard ratio, 0.108 ; $95 \%$ confidential interval (CI), 0.029-0.401; P=0.001; Table III].

Univariate analysis and multivariate statistics for intrahepatic distant recurrence and TFS rate. A univariate analysis using the log-rank test revealed that the intrahepatic distant recurrence and TFS rate were only positively associated with RFA/TACE (data not shown). A multivariate analysis using a Cox proportional hazard model, including age and sex, for six markers (ALT, $\gamma$-GTP, Plt, DCP, ablation margin and RFA/TACE) selected based on $\mathrm{P}<0.500$ in univariate analysis, revealed that RFA/TACE was the only independent risk factor associated with intrahepatic distant recurrence factors (odds ratio, 0.467; 95\% CI, 0.225-0.973; $\mathrm{P}=0.042$; Table IV) and for seven markers

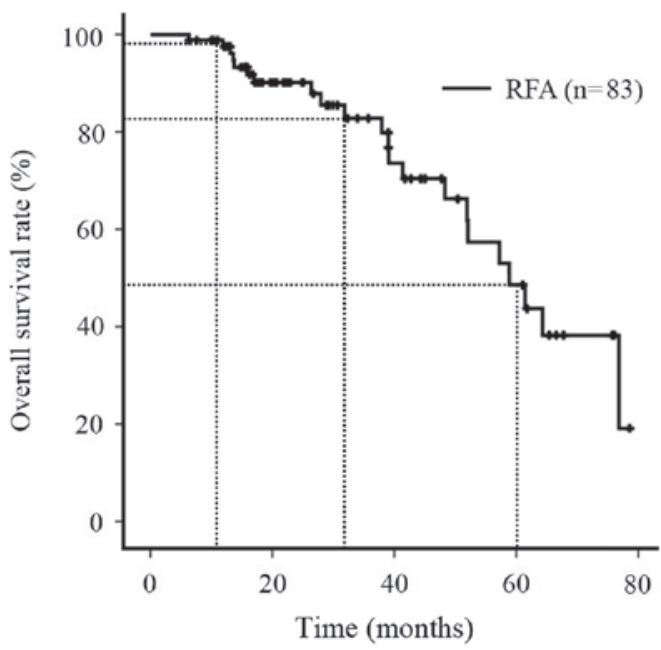

Figure 1. Overall survival rate of 83 patients with early hepatocellular carcinoma treated with RFA alone. The overall survival rates during the follow-up period were $97.5 \%$ at 1 year, $82.8 \%$ at 3 years and $48.6 \%$ at 5 years. RFA, radiofrequency ablation.

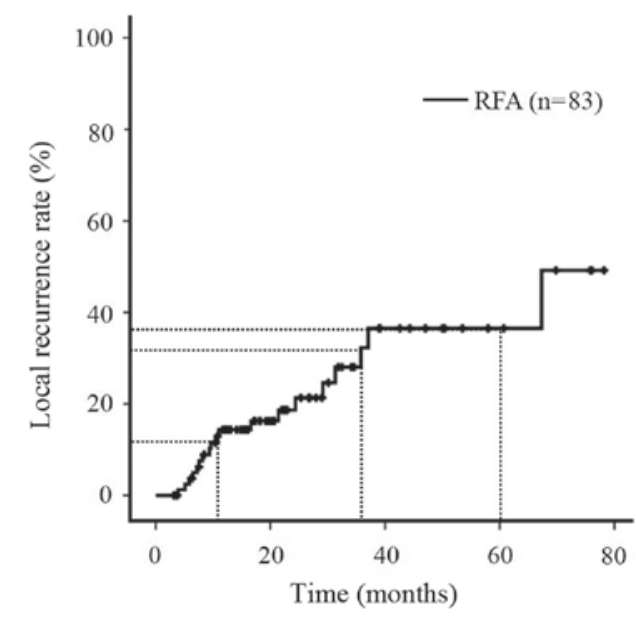

Figure 2. Local recurrence rate of 83 patients with early hepatocellular carcinoma treated with RFA alone. The local recurrence rates were $14.3 \%$ at 1 year, $32.3 \%$ at 3 years and $36.5 \%$ at 5 years. RFA, radiofrequency ablation.

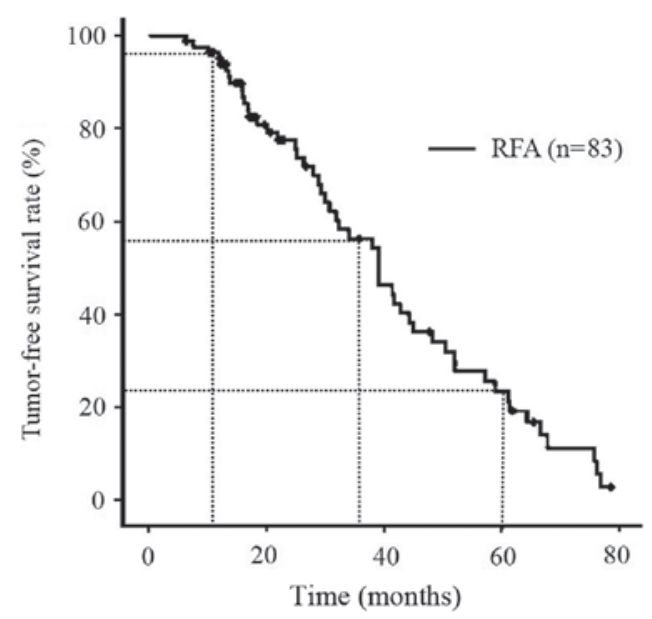

Figure 3. Tumor-free survival rate of 83 patients with early hepatocellular carcinoma treated with RFA alone. The tumor-free survival rates were $95.1 \%$ at 1 year, $56.3 \%$ at 3 years and $23.4 \%$ at 5 years. RFA, radiofrequency ablation. 
Table II. Clinical characteristics of 83 patients with early, solitary and hypervascular hepatocellular carcinoma.

\begin{tabular}{|c|c|c|c|}
\hline Factor & RFA/TACE group, $\mathrm{n}$ & RFA alone group, $\mathrm{n}$ & P-value \\
\hline Total no. of patients & 56 & 27 & \\
\hline Age, years & $73.0(45-91)$ & $73.0(57-89)$ & 0.218 \\
\hline Sex, male/female & $30 / 26$ & $11 / 16$ & 0.350 \\
\hline Virus marker, HBV/HCV/NBNC & $6 / 39 / 11$ & $1 / 21 / 5$ & 0.538 \\
\hline Prothrombin time, $\%$ & $82.8(35-110)$ & $83.4(51-101)$ & 0.857 \\
\hline Total bilirubin, mg/dl & $0.9(0.3-2.6)$ & $1.1(0.4-2.5)$ & 0.205 \\
\hline Serum albumin, $\mathrm{g} / \mathrm{dl}$ & $3.6(2.8-4.8)$ & $3.6(2.4-4.9)$ & 0.934 \\
\hline AST, IU/1 & $44.0(19-110)$ & $45.0(17-84)$ & 0.613 \\
\hline ALT, IU/1 & $35.0(13-116)$ & $31.0(9-84)$ & 0.644 \\
\hline$\gamma$-GTP, IU 1 & $81.7(34.7-110)$ & $83.4(51-101)$ & 0.166 \\
\hline Platelet count, $\mathrm{x} 10^{4} / \mu 1$ & $9.5(3.3-27.6)$ & $9.3(3.1-19.8)$ & 0.637 \\
\hline AFP, ng/ml & $13.7(1.5-7,931)$ & $13.4(3.6-8,609)$ & 0.996 \\
\hline $\mathrm{DCP}, \mathrm{mAU} / \mathrm{ml}$ & $33.5(12-9,455)$ & $33.0(8.0-2,314)$ & 0.656 \\
\hline Previous treatment, $-/+$ & $29 / 27$ & $12 / 15$ & 0.641 \\
\hline Intrahepatic recurrence, $-/+$ & $29 / 27$ & $12 / 15$ & 0.641 \\
\hline Tumor size, mm & $17.8(10-30)$ & $13.3(6-30)$ & 0.004 \\
\hline Ablated size, $\mathrm{mm}$ & $31.0(19-52)$ & $28.5(20-48)$ & 0.527 \\
\hline
\end{tabular}

Data are presented as geometric median (range) or number. RFA, radiofrequency ablation; TACE, transarterial chemoembolization; RFA/TACE, RFA combined with TACE; HBV, hepatitis B virus; HCV, hepatitis C virus; NBNC, HBV (-) and HCV (-); AST, aspartate transaminase; ALT, alanine aminotransferase; $\gamma$-GTP, $\gamma$-glutamyl transpeptidase; AFP, $\alpha$-fetoprotein; DCP, des- $\gamma$-carboxy prothrombin. Previous treatment included treatment surgery, RFA and TACE.

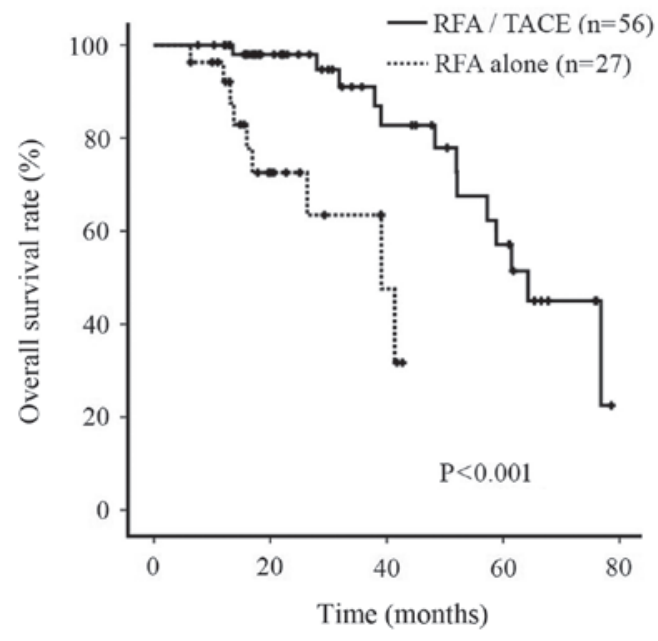

Figure 4. Overall survival rate of 83 patients with early hepatocellular carcinoma treated with RFA/TACE vs. RFA alone. RFA, radiofrequency ablation; RFA/TACE, RFA combined with transarterial chemoembolization.

(total bilirubin, AST, ALT, $\gamma$-GTP, tumor size, ablated margin and RFA/TACE) selected based on $\mathrm{P}<0.500$ in univariate analysis, revealed that RFA/TACE was the only independent risk factor associated with tumor-free survival factors (odds ratio, 0.452; 95\% $\mathrm{CI}, 0.224-0.842 ; \mathrm{P}=0.012$; Table IV).

\section{Discussion}

In the treatment algorithm for HCC, based on the Japan Society of Hepatology consensus, liver resection or localized therapy

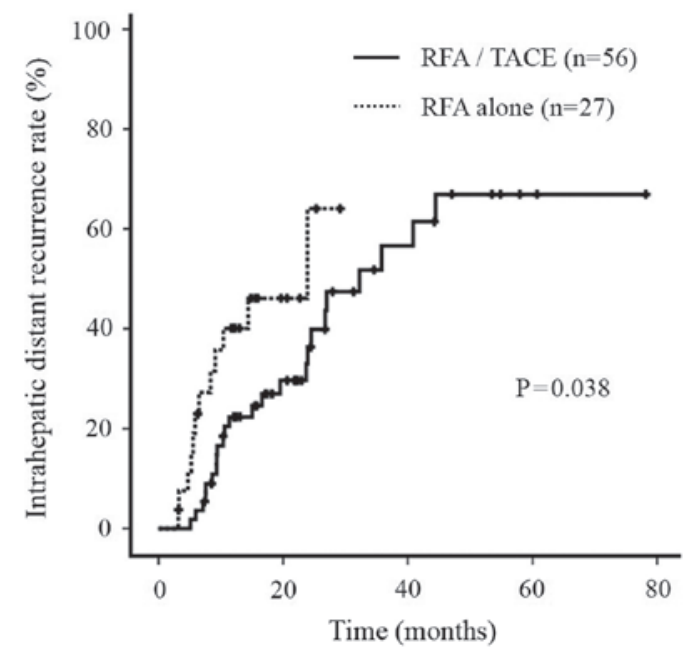

Figure 5. Progression rate of other intrahepatic tumors in 83 patients with early hepatocellular carcinoma treated with RFA/TACE vs. RFA alone. RFA, radiofrequency ablation; RFA/TACE, RFA combined with transarterial chemoembolization.

is recommended based on remaining liver function (4). In contrast, in the treatment algorithm based on the BCLC staging system, solitary HCCs $<2 \mathrm{~cm}$ in diameter were classified as very early stage (stage 0 ) (3). The 5-year OS rate of patients undergoing liver resection and liver transplant was reported as $80-90 \%$, while it was $70 \%$ in those undergoing localized ablation $(6,17,18)$. In addition, patients with single tumors $>2 \mathrm{~cm}$ or three nodules $<3 \mathrm{~cm}$ in diameter were classified as early HCC (BCLC stage A) with a 5 year OS rate of $50-70 \%$ for 
Table III. Evaluation of the prognostic factors in the early, solitary and hypervascular hepatocellular carcinoma cases.

\begin{tabular}{|c|c|c|c|c|c|}
\hline \multirow[b]{2}{*}{ Factor (categories) } & \multicolumn{2}{|c|}{ Univariate } & \multicolumn{3}{|c|}{ Multivariate } \\
\hline & $\mathrm{n}=83$ & $\mathrm{P}$-value & HR & $(95 \% \mathrm{CI})$ & P-value \\
\hline Age $(<70 / \geq 70$ years $)$ & $28 / 55$ & 0.008 & 0.459 & $0.162-1.302$ & 0.143 \\
\hline Sex (male/female) & $41 / 42$ & 0.601 & 0.601 & $0.213-1.700$ & 0.337 \\
\hline Total bilirubin, $\mathrm{mg} / \mathrm{dl}(<1.0 / \geq 1.0)$ & $37 / 46$ & 0.066 & & & \\
\hline $\mathrm{AST}, \mathrm{IU} / 1(<50 / \geq 50)$ & $49 / 34$ & 0.003 & & & \\
\hline ALT, IU/1 $(<40 / \geq 40)$ & $48 / 35$ & 0.006 & & & \\
\hline$\gamma$-GTP, IU/l $(<45 / \geq 45)$ & $54 / 29$ & 0.162 & & & \\
\hline Serum albumin, g/dl $(<3.6 / \geq 3.6)$ & $47 / 36$ & 0.102 & & & \\
\hline Platelet count, $\times 10^{4} / \mu 1(<10 / \geq 10)$ & $47 / 36$ & 0.05 & & & \\
\hline Prothrombin time, $\%(<85 / \geq 85)$ & $48 / 35$ & 0.134 & & & \\
\hline AFP, ng/ml $(<15 / \geq 15)$ & $43 / 40$ & 0.291 & & & \\
\hline $\mathrm{DCP}, \mathrm{mAU} / \mathrm{ml}(<35 / \geq 35)$ & $44 / 39$ & 0.400 & & & \\
\hline Tumor size, $\mathrm{mm}(<20 / \geq 20)$ & $54 / 29$ & 0.064 & & & \\
\hline Ablated margin, $\mathrm{mm}(<5 / \geq 5)$ & $59 / 24$ & 0.496 & & & \\
\hline RFA/TACE (-/+) & $27 / 56$ & $<0.001$ & 0.108 & $0.029-0.401$ & 0.001 \\
\hline
\end{tabular}

Univariate analysis was conducted by employing the log-rank test. Multivariate analysis was conducted by employing the Cox proportional hazards model. AST, aspartate transaminase; ALT, alanine aminotransferase; $\gamma$-GTP, $\gamma$-glutamyl transpeptidase; AFP, $\alpha$-fetoprotein; DCP, des- $\gamma$-carboxy prothrombin; RFA, radiofrequency ablation; TACE, transarterial chemoembolization; RFA/TACE, RFA combined with TACE; HR, hazard ratio; CI, confidence interval.

Table IV. Multivariate statistics for the intrahepatic distant recurrence and tumor-free survival factors in the early, solitary and hypervascular hepatocellular carcinoma cases.

\begin{tabular}{lccc}
\hline RFA/TACE (-/+) & OR & $95 \%$ CI & P-value \\
\hline $\begin{array}{l}\text { The intrahepatic distant } \\
\text { recurrence }\end{array}$ & 0.467 & $0.225-0.973$ & 0.042 \\
\begin{tabular}{l} 
The tumor-free survival \\
\hline
\end{tabular} & 0.452 & $0.224-0.842$ & 0.012 \\
\hline
\end{tabular}

Multivariate analysis was conducted by employing the Cox proportional hazards model. OR, odds ratio; CI, confidence interval; RFA, radiofrequency ablation; TACE, transarterial chemoembolization; RFA/TACE, RFA combined with TACE.

liver resection, liver transplant and localized ablation $(16,19)$. Although this was a retrospective study, to reduce bias, only subjects suspected of having moderately-differentiated liver cancer identified via imaging as single hypervascular tumors were included. As a result, patients with single HCC tumors $<3 \mathrm{~cm}$ that exhibited early-phase staining were enrolled, and it was demonstrated that the rates of overall and tumor-free survival were not inferior to previous studies (20). In addition, results were obtained during long-term follow-up of over 5 years. In terms of TFS, the 5-year survival rate was slightly low at 23.4\%; however, multiple patients with HCC also had concomitant $\mathrm{HCV}$ infections. Therefore, even if local factors were controlled, there may still have been patients who relapsed.

At Kagoshima University Medical and Dental Hospital, Kagoshima Teishin Hospital and Kagoshima City Hospital,

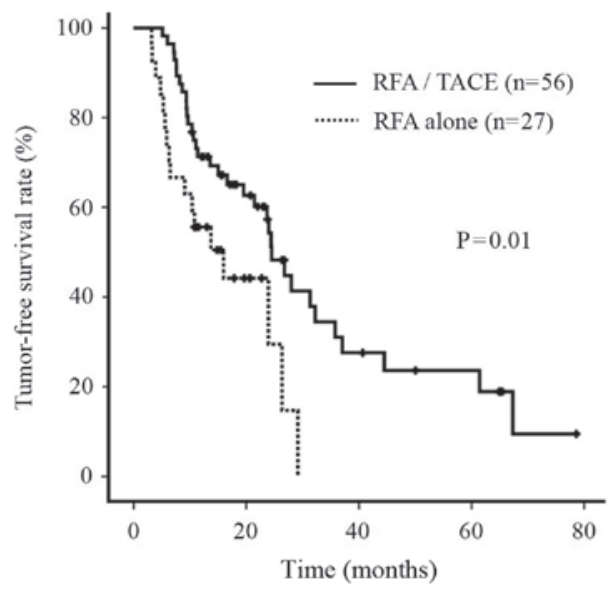

Figure 6. During the follow-up period, the tumor-free survival rate of patients treated with RFA/TACE was significantly improved compared with that of patients treated with RFA alone. RFA, radiofrequency ablation; RFA/TACE, RFA combined with transarterial chemoembolization.

RFA is often performed as a curative therapy following TACE in the tumor-bearing area. The main reasons for recommending RFA/TACE are as follows: When performing TACE in the tumor-bearing area, an antitumor effect is expected in the primary lesion as well as the surrounding area; lipiodol accumulates in the tumor, serving as a marker when performing the RFA, and the post-treatment identification of the ablation area is easier (21); expansion of the ablation area is expected in areas in which lipiodol accumulates following TACE, making it appropriate for slightly larger HCC (22); and the combination of TACE and RFA results in improved local 
control even when lesions occur on the surface of the liver or near blood vessels.

Multiple studies have been conducted on RFA/TACE, and a systematic review (23) performed a meta-analysis of eight clinical trials (24-30). However, the majority of these studies examined HCC tumors $>3 \mathrm{~cm}$ in diameter, and only one examined small HCCs (28). The meta-analysis revealed that rate of local tumor progression, OS, local progression-free survival and event-free survival were not significantly different between the combination therapy and RFA alone. There are no details regarding the inclusion criteria aside from the fact that the analyzed trials enrolled patients with HCC with three tumors that were $<3 \mathrm{~cm}$ in diameter, which differs from the present study. Kim et al (10) also published retrospective data, but they reported on a small number of early-stage HCCs of 2-3 cm diameter. Furthermore, although RFA/TACE demonstrated a higher rate of local progression-free survival and event-free survival compared with RFA alone, no significant difference in OS was observed, which contradicts the results of the present study. Finally, multivariate analysis was not performed in this previous study, and therefore it is possible to consider the present study as the first to demonstrate that RFA/TACE is an independent determining factor of prognosis and relapse.

Nakashima et al (31) investigated 209 nodules $<3 \mathrm{~cm}$ in diameter that were surgically resected, and revealed that 'single nodular type with extranodular growth' and 'confluent multinodular type' demonstrated higher frequencies of portal vein invasion and intrahepatic metastases compared with 'single nodular type'. In addition, they reported that among 149 metastatic lesions, the distance from the primary tumor was $\leq 10 \mathrm{~mm}$ in $118(79.2 \%)$ cases. Furthermore, Nishikawa et al (21) proposed a method for grading HCC based on ablative margins and its use in predicting local recurrence. For Grade A (absolutely curative) tumors the ablative margin around the tumor was $>5 \mathrm{~mm}$, for Grade B (relatively curative) the margin was $<5 \mathrm{~mm}$, for Grade $\mathrm{C}$ (relatively non-curative) there was no complete ablative margin although no residual tumor was apparent, and for Grade D (absolutely non-curative) the tumor had not been entirely ablated. The cumulative localized rate of recurrence was significantly improved for Grades A and B compared with Grades C and D, and it was extremely important that the ablation range (cauterization margin) was adequately achieved by RFA. In the present study, the average tumor size was larger in the RFA/TACE group compared with the RFA-only group. In addition, multiple patients had inadequate tumor and ablation margins following RFA alone. Despite these drawbacks, favorable results for cumulative survival rate, intrahepatic tumor progression rate and tumor-free survival were obtained, indicating that even small HCCs $<3 \mathrm{~cm}$ in diameter possess microscopic disseminated disease, depending on gross morphology. Patients with cirrhosis commonly have fine arterioportal shunts and hepatofugal blood flow (32). Anticancer drugs administered during TACE are thought to be washed out immediately, except for Miripla (33), but these drugs are likely to be carried in blood that flows outside subsegments or the entire liver, via the same hemodynamic route used in the dissemination of liver cancer cells. Anticancer drugs may suppress recurrence of micro-level dissemination of cells during TACE that cannot be detected at the macro level $(34,35)$. These observations supported the idea that treatment with TACE in the tumor-bearing zone not only affects the primary lesion, but also suppresses local and ectopic recurrences.

There are several limitations to the present study. First, it included a small number of patients. Second, there may be slight biases in treatment approaches and patient selection due to this being a multicenter study. Third, the study design was not a prospective randomized controlled trial. Fourth, propensity matching was not performed for either group despite the retrospective nature of the study. Tumor size was significantly larger in patients treated with RFA/TACE, which was an analytical disadvantage; however, no significant differences in baseline characteristics of patients were observed. Propensity matching may have eliminated the biases between the groups, but would have reduced the usable patient population, making adequate analysis difficult. Furthermore, for more rigorous analysis, a randomized controlled trial is desirable.

In conclusion, the present study revealed that treatment with RFA/TACE improved prognosis, the rate of intrahepatic recurrence and tumor-free survival compared with RFA alone. The present study therefore demonstrated that RFA/TACE is effective in patients with small HCC. However, a large-scale randomized controlled trial is required to compare the results with those obtained from therapy using RFA alone.

\section{References}

1. Torre LA, Bray F, Siegel RL, Ferlay J, Lortet-Tieulent J and Jemal A: Global cancer statistics, 2012. CA Cancer J Clin 65: 87-108, 2015.

2. Bruix J and Sherman M; American Association for the Study of Liver Diseases: Management of hepatocellular carcinoma: An update. Hepatology 53: 1020-1022, 2011.

3. European Association For The Study Of The Liver; European Organisation For Research And Treatment Of Cancer: EASL-EORTC clinical practice guidelines: Management of hepatocellular carcinoma. J Hepatol 56: 908-943, 2012.

4. Kudo M, Matsui O, Izumi N, Iijima H, Kadoya M, Imai Y, Okusaka T, Miyayama S, Tsuchiya K, Ueshima K, et al: JSH consensus-based clinical practice guidelines for the management of hepatocellular carcinoma: 2014 Update by the Liver Cancer Study Group of Japan. Liver Cancer 3: 458-468, 2014.

5. Lafaro KJ, Demirjian AN and Pawlik TM: Epidemiology of hepatocellular carcinoma. Surg Oncol Clin N Am 24: 1-17, 2015.

6. Takayama T, Makuuchi M, Hirohashi S, Sakamoto M, Yamamoto J, Shimada K, Kosuge T, Okada S, Takayasu K and Yamasaki S: Early hepatocellular carcinoma as an entity with a high rate of surgical cure. Hepatology 28: 1241-1246, 1998.

7. Hong SN, Lee SY, Choi MS, Lee JH, Koh KC, Paik SW, Yoo BC, Rhee JC, Choi D, Lim HK, et al: Comparing the outcomes of radiofrequency ablation and surgery in patients with a single small hepatocellular carcinoma and well-preserved hepatic function. J Clin Gastroenterol 39: 247-252, 2005.

8. Ueno M, Hayami S, Shigekawa Y, Kawai M, Hirono S, Okada K, Tamai H, Shingaki N, Mori Y, Ichinose M and Yamaue H: Prognostic impact of surgery and radiofrequency ablation on single nodular HCC $5 \mathrm{~cm}$ : Cohort study based on serum HCC markers. J Hepatol 63: 1352-1359, 2015.

9. Nakai M, Sato M, Sahara S, Takasaka I, Kawai N,MinamiguchiH, Tanihata H, Kimura M and Takeuchi N: Radiofrequency ablation assisted by real-time virtual sonography and CT for hepatocellular carcinoma undetectable by conventional sonography. Cardiovasc Intervent Radiol 32: 62-69, 2009.

10. Kim JW, Kim JH, Won HJ, Shin YM, Yoon HK, Sung KB and Kim PN: Hepatocellular carcinomas $2-3 \mathrm{~cm}$ in diameter: Transarterial chemoembolization plus radiofrequency ablation vs. Radiofrequency ablation alone. Eur J Radiol 81: e189-e193, 2012.

11. Llovet JM, Brú C and Bruix J: Prognosis of hepatocellular carcinoma: The BCLC staging classification. Semin Liver Dis 19: 329-338, 1999. 
12. Llovet JM, Di Bisceglie AM, Bruix J, Kramer BS, Lencioni R, Zhu AX, Sherman M, Schwartz M, Lotze M, Talwalkar J, et al: Design and endpoints of clinical trials in hepatocellular carcinoma. J Natl Cancer Inst 100: 698-711, 2008.

13. Seldinger SI: Catheter replacement of the needle in percutaneous arteriography; a new technique. Acta Radiol 39: 368-376, 1953.

14. Albers I, Hartmann H, Bircher J and Creutzfeldt W: Superiority of the Child-Pugh classification to quantitative liver function tests for assessing prognosis of liver cirrhosis. Scand J Gastroenterol 24: 269-276, 1989.

15. Novák $\mathbf{J}$ and Fabian P: Comments on the TNM classification of malignant tumours-7th edition. Klin Onkol 24: 149-150, 2011 (In Czech).

16. Llovet JM and Bruix J: Novel advancements in the management of hepatocellular carcinoma in 2008. J Hepatol 48 (Suppl 1): S20-S37, 2008.

17. Roayaie S, Blume IN, Thung SN, Guido M, Fiel MI, Hiotis S, Labow DM, Llovet JM and Schwartz ME: A system of classifying microvascular invasion to predict outcome after resection in patients with hepatocellular carcinoma. Gastroenterology 137 850-855, 2009

18. Livraghi T, Meloni F, Di Stasi M, Rolle E, Solbiati L, Tinelli C and Rossi S: Sustained complete response and complications rates after radiofrequency ablation of very early hepatocellular carcinoma in cirrhosis: Is resection still the treatment of choice? Hepatology 47: 82-89, 2008.

19. Arii S, Yamaoka Y, Futagawa S, Inoue K, Kobayashi K, Kojiro M, Makuuchi M, Nakamura Y, Okita K and Yamada R: Results of surgical and nonsurgical treatment for small-sized hepatocellular carcinomas: A retrospective and nationwide survey in Japan. The Liver Cancer Study Group of Japan. Hepatology 32: 1224-1229, 2000.

20. Waki K, Aikata H, Katamura Y, Kawaoka T, Takaki S, Hiramatsu A, Takahashi S, Toyota N, Ito K and Chayama K Percutaneous radiofrequency ablation as first-line treatment for small hepatocellular carcinoma: results and prognostic factors on long-term follow up. J Gastroenterol Hepatol 25: 597-604, 2010.

21. Nishikawa H, Inuzuka T, Takeda H, Nakajima J, Sakamoto A Henmi S, Matsuda F, Eso Y, Ishikawa T, Saito S, et al: Percutaneous radiofrequency ablation therapy for hepatocellular carcinoma: A proposed new grading system for the ablative margin and prediction of local tumor progression and its validation. J Gastroenterol 46: 1418-1426, 2011.

22. Yamakado K, Nakatsuka A, Akeboshi M, Shiraki K, Nakano T and Takeda K: Combination therapy with radiofrequency ablation and transcatheter chemoembolization for the treatment of hepatocellular carcinoma: Short-term recurrences and survival. Oncol Rep 11: 105-109, 2004.

23. Chen QW, Ying HF, Gao S, Shen YH, Meng ZQ, Chen H, Chen Z and Teng WJ: Radiofrequency ablation plus chemoembolization versus radiofrequency ablation alone for hepatocellular carcinoma: A systematic review and meta-analysis. Clin Res Hepatol Gastroenterol 40: 309-314, 2016.

24. Peng ZW, Zhang YJ, Chen MS, Xu L, Liang HH, Lin XJ, Guo RP, Zhang YQ and Lau WY: Radiofrequency ablation with or without transcatheter arterial chemoembolization in the treatment of hepatocellular carcinoma: A prospective randomized trial. J Clin Oncol 31: 426-432, 2013.
25. Peng ZW, Zhang YJ, Liang HH, Lin XJ, Guo RP and Chen MS Recurrent hepatocellular carcinoma treated with sequential transcatheter arterial chemoembolization and RF ablation versus RF ablation alone: A prospective randomized trial. Radiology 262: 689-700, 2012.

26. Morimoto M, Numata K, Kondou M, Nozaki A, Morita S and Tanaka K: Midterm outcomes in patients with intermediate-sized hepatocellular carcinoma: A randomized controlled trial for determining the efficacy of radiofrequency ablation combined with transcatheter arterial chemoembolization. Cancer 116 : 5452-5460, 2010

27. Yang W, Chen MH, Wang MQ, Cui M, Gao W, Wu W, Wu JY, Dai $Y$ and Yan K: Combination therapy of radiofrequency ablation and transarterial chemoembolization in recurrent hepatocellular carcinoma after hepatectomy compared with single treatment. Hepatol Res 39: 231-240, 2009.

28. Shibata T, Isoda H, Hirokawa Y, Arizono S, Shimada K and Togashi K: Small hepatocellular carcinoma: Is radiofrequency ablation combined with transcatheter arterial chemoembolization more effective than radiofrequency ablation alone for treatment? Radiology 252: 905-913, 2009.

29. Shen SQ, Xiang JJ, Xiong CL, Wu SM and Zhu SS: Intraoperative radiofrequency thermal ablation combined with portal vein infusion chemotherapy and transarterial chemoembolization for unresectable HCC. Hepatogastroenterology 52: 1403-1407, 2005.

30. Zhang Z, Wu M, Chen H, Chen D and He J: Percutaneous radiofrequency ablation combined with transcatheter arterial chemoembolization for hepatocellular carcinoma. Zhonghua Wai Ke Za Zhi 40: 826-829, 2002 (In Chinese).

31. Nakashima Y, Nakashima O, Tanaka M, Okuda K, Nakashima M and Kojiro M: Portal vein invasion and intrahepatic micrometastasis in small hepatocellular carcinoma by gross type. Hepatol Res 26: 142-147, 2003.

32. Kim TK, Choi BI, Han JK, Chung JW, Park JH and Han MC: Nontumorous arterioportal shunt mimicking hypervascular tumor in cirrhotic liver: Two-phase spiral CT findings. Radiology 208: 597-603, 1998.

33. Imai Y, Chikayama T, Nakazawa M, Watanabe K, Ando S, Mizuno Y, Yoshino K, Sugawara K, Hamaoka K, Fujimori K, et al: Usefulness of miriplatin as an anticancer agent for transcatheter arterial chemoembolization in patients with unresectable hepatocellular carcinoma. J Gastroenterol 47: 179-186, 2012.

34. Wang YX, De Baere T, Idée JM and Ballet S: Transcatheter embolization therapy in liver cancer: An update of clinical evidences. Chin J Cancer Res 27: 96-121, 2015.

35. Fang ZT, Zhang W, Wang GZ, Zhou B, Yang GW, Qu XD, Liu R, Qian S, Zhu L, Liu LX, et al: Circulating tumor cells in the central and peripheral venous compartment-assessing hematogenous dissemination after transarterial chemoembolization of hepatocellular carcinoma. Onco Targets Ther 7: 1311-1318, 2014. 